
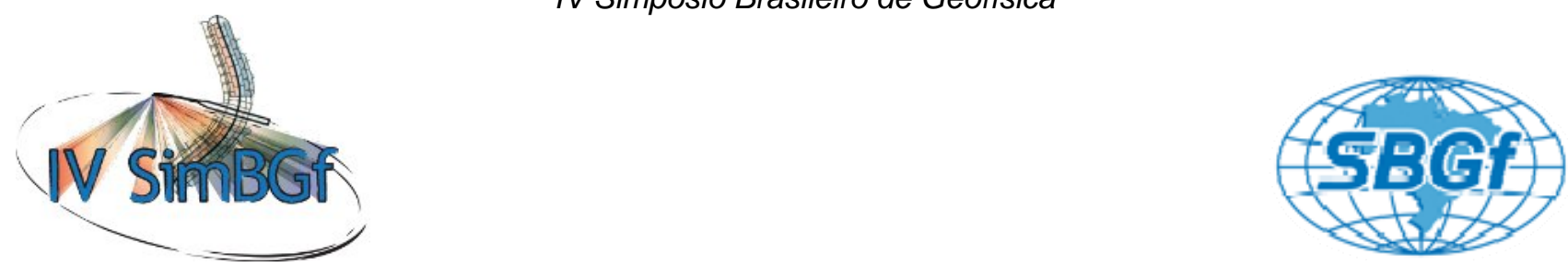

\title{
Modelamento gravimétrico aplicado a exploração das mineralizações de ferro do Projeto Porteirinha, Grupo Macaúbas - Neoproterozóico, norte de Minas Gerais-Brasil.
}

Cristiane Moura* (Vale S.A), Flávio Freitas-Silva (Vale S.A), Florivaldo Sena (Vale S.A), Cantidiano Freitas (Vale S.A), Marco Antônio Braga (Vale S.A).

Copyright 2010, SBGf - Sociedade Brasileira de Geofísica

Este texto foi preparado para a apresentação no IV Simpósio Brasileiro de Geofísica Brasília, 14 a 17 de novembro de 2010. Seu conteúdo foi revisado pelo Comite Técnico do IV SimBGf, mas não necessariamente representa a opinião da SBGf ou de seus associados. É proibida a reprodução total ou parcial deste material para propósitos comerciais sem prévia autorização da SBGf.

\section{Abstract}

The modeling of airgravity gradiometry data of the Porteirinha Iron Project allowed to improve the structural framework and individualization of lithostratigraphyc units and mineralized rocks. Applying only routine procedures for terrain corrections utilizing topographic regional data could be established the main regional ore controls. Airgravity modeling on a detail scale required a more robust terrain corrections based on aerial laser topography, which allowed good assessment of the geometry of the mineralized layers and their host rocks. The gravity model was also able to predict the areas of higher grade ores as well as possible changes in the geometry of the mineralized levels caused by the overprinting of deformation. The low density contrast between the ores and the host rocks demanded careful integration between gravity data, field geology observations, density and iron grades, which were obtained in boreholes. In order to evaluate accuracy of the gravity model the total field magnetic survey was compared to magnetic susceptibility of each litotype suggested by the gravity Tzz curve resulting in reasonable coincidence between measured and calculated magnetic curve. Therefore, the use of airgravity modeling in the Porterinha Project presented very good results on mapping covered iron ores, even predicting higher grade zones which were confirmed by drill holes intersections, showing that it can be a very important technique to be used in iron exploration programs.

\section{Introdução}

Os depósitos de ferro e manganês do Projeto Porteirinha, norte do Estado de Minas Gerais (Figura 01) têm sido pesquisados desde a década de 70 (Schobbenhaus, 1972ab, Vilela et al. 1978, Viveiros et al. 1978ab, Viveiros et al. 1979, Vilela 1986). A área aqui abordada faz parte do Projeto Porteirinha, o qual atualmente soma recursos de aproximadamente 5Bt @ 35\%Fe (Correia et al. 2009). Os principais tipos de minérios de ferro da região do Projeto Porteirinha são constituídos por diamictitos ferruginosos e formações ferríferas granulares bandadas - Gifs (Clout \& Simonson 2005, Bekker et al. 2010) subordinadas, encaixadas e geneticamente ligadas a unidades sedimentares glaciogênicas pertencente ao Grupo Macaúbas (Karfunkel et al. 1985, Noce et al. 1997) sendo classificados como minérios de ferro do tipo Rapitan (Raptan type iron ore formation - Clout \& Simonson 2005, Bekker et al. 2010, Young 1976, Klein 1993). Os depósitos de ferro glaciogênicos do norte de Minas Gerais apresentam grande volume, sendo que o principal tipo de minério de ferro corresponde a diamictitos ferruginosos com teores de ferro entre $30 \%$ e $50 \%$, em meios aos quais ocorrem zonas de minérios maciços com teores acima de $60 \%$ de ferro (Corrêia et al. 2009).

As unidades glaciogênicas que compõem o Grupo Macaúbas se caracterizam por uma grande variação faciológica (cf. revisão e bibliografia em Uhlein et al. 1999; Roque et al. 1997; Pedrosa-Soares \& Oliveira 1997), que aliado às deformações e as condições nem sempre favoráveis de afloramentos dificultam os trabalhos de exploração dos minérios de ferro, desta maneira, a utilização de ferramentas geofísicas tem sido necessários no auxilio aos trabalhos de geologia.

Toda a região norte do Estado de Minas Gerais foi coberta por levantamento aerogeofísico magnético e gamaespectrométrico (CODEMIG 2006), e com a retomada dos trabalhos na região na área do Projeto Porteirinha - Vale S.A foram também adquiridos dados geofísicos de aerogradiometria gravimétrica FTG (Full Tensor Gradiometry - Air-FTG 3D), sendo o objetivo deste trabalho apresentar os resultados e a aplicação do modelamento gravimétrico como suporte aos trabalhos de exploração mineral.

\section{Metodologia / Problema Investigado}

Os procedimentos para a caracterização das fontes geradoras das anomalias gravimétricas, que estão representadas pelos produtos de modelagem, englobam o processamento e interpretação do conjunto de informações geofísicas de aerogradiometria gravimétrica, integradas as informações de mapeamento geológico, aeromagnetometria e dados testemunhos de sondagem, que compreendem as descrições dos litotipos, os resultados geoquímicos e ensaio de densidade. Para a realização dos trabalhos foi utilizado o módulo GMSYS 2D do aplicativo Geosoft 7.2. A Tabela 01 apresenta os 
parâmetros estatísticos de densidade dos litotipos do Projeto Porteirinha.

Os trabalhos de modelagem aqui abordados referem-se aos levantamentos executados nos alvos Faixa Oeste, Faixa Leste, Peixe Bravo, Morro Grande e Serra do Capim que integram o Projeto Porteirinha (Figura 01).

A modelagem 2D dos dados geofísicos de aerogradiometria gravimétrica buscou a identificação em subsuperfície dos diferentes litotipos em função do contraste de densidade entre as rochas, que em conjunto com dados geológicos, geoquímicos e de sondagem permitiram fornecer informações precisas aos trabalhos de exploração, como uma melhor adequação das malhas de sondagem além de auxiliar na interpretação da geometria dos corpos de minério e da estimativa de recursos.

\begin{tabular}{|l|c|c|c|c|c|}
\hline Tabela 01 - Parâmetros estatísticos de densidade $\left(\mathrm{g} / \mathrm{cm}^{3}\right)$ dos litotipos do Projeto Porteirinha. \\
\hline & DHC & DHF & CAN & DIC & DIC-SER \\
\hline Numero de amostras & 141 & 157 & 16 & 45 & 5 \\
\hline Mínimo & 2.41 & 1.93 & 2.09 & 2.38 & 2.69 \\
\hline Maximo & 4.40 & 4.51 & 3.82 & 3.57 & 2.96 \\
\hline Média & 3.33 & 3.34 & 2.76 & 2.91 & 2.83 \\
\hline Mediana & 3.25 & 3.37 & 2.60 & 2.92 & 2.83 \\
\hline Variância & 0.11 & 0.26 & 0.28 & 0.07 & 0.01 \\
\hline Desvio padrão & 0.33 & 0.51 & 0.53 & 0.26 & 0.10 \\
\hline $\begin{array}{l}\text { DHC = Diamictito Hematítico Compacto; DHF = Diamictito Hematítico Frível; CAN = Canga; DIC = Diamictito } \\
\text { Cinza; DIC-SER = Diamictito Sericitico }\end{array}$ \\
\hline
\end{tabular}

\section{Resultados}

As unidades litológicas que compõem o depósito de ferro de Porteirinha apresentam baixos contrastes de densidade (Tabela 01), desta forma para seu modelamento os dados devem ser corrigidos exigindo levantamentos topográficos de alta precisão e entendimento dos principais condicionantes da geometria do corpo de minério. A nível regional as mineralizações apresentam um controle litoestratigráfico comportando-se como corpos tabulares, esta geometria é modificada pelo padrão de deformação, condicionada a dobras inversas regionais $\left(D_{1}\right)$, aos dobramentos/flexuras cruzados de grande amplitude $\left(D_{2 a}\right.$ e $\left.\quad D_{2 b}\right)$ e aos falhamentos extensionais tardios.

De forma a se ter um melhor controle da continuidade das zonas mineralizadas, em adição às feições geológicas levantadas em mapeamentos e sondagens, foram modelados os dados de teores de ferro $e$ densidade (Figuras 02, 03 e 04). A análise destes produtos, de acordo com as observações de campo, sugere que as regiões de mais elevados teores estão condicionadas a charneiras sinclinais $D_{1}$ de primeira e segunda ordem (saddle reefs), independentemente se localizadas em flancos inversos (Figuras $5 a, b, c)$ ou em flancos normais (Figura $5 d$ ) das dobras regionais.

As principais modificações na geometria do minério, que impõem maiores dificuldades a modelagem são ocasionadas por dobramentos tardios $\left(D_{2 a}\right.$ e $\left.D_{2 b}\right)$. Os falhamentos extensionais finais, embora possam deslocar e/ou bascular grandes blocos, não modificam a geometria interna das camadas.
Na Figura 5 são apresentados exemplos dos produtos finais alcançados com a modelagem dos dados de aerogradiometria gravimétrica. Como podem ser observado nesta figura os ajustes entre as curvas observadas e as curvas calculadas é bastante satisfatórios e coerentes com as demais feições, como o arranjo estratigráfico, o padrão de deformação e ao ajuste da modelagem dos dados magnéticos.

Quanto à distribuição dos litotipos nos diferentes alvos, destaca-se a caracterização das camadas de diamictitos de mais elevada densidades, nomeados $\mathrm{DH} 1$ na Figura 05, por vezes compondo zonas de brechas, sugerindo tratar-se não somente de uma feição primária, mas de enriquecimentos condicionados por deslizamentos flexurais entre camadas As zonas com presença de hematita friável e cangas estruturais (Figura 05) embora sejam em grande parte controladas por processos supergênicos ocorrem preferencialmente em zonas de charneiras (sadlle reefs) e ao longo de planos axiais de sinclinais $D_{1}$.

\section{Discussão e Conclusões}

O levantamento aerogradiométrico gravimétrico na região do Projeto Porteirinha com as correções de terreno utilizando apenas produtos regionais como cartas 1:100.000 ou dados SRTM (Shuttle Radar Terrain Model) proporcionam a caracterização do arcabouço regional das ocorrências de minério de ferro do Projeto Porteirinha, complementando e refinando os dados de levantamentos aeromagnéticos.

Para a modelagem é necessário a correção de terreno com dados topográficos mais precisos, neste sentido os aerolevantamentos de topografia a laser mostraram-se satisfatórios para individualização dos diferentes litotipos, bem como da identificação e previsão de zonas enriquecidas em ferro.

As correções de terreno executadas com os dados de topografia a laser foram satisfatórias para as regiões topograficamente elevadas, entretanto, não foi suficiente para eliminar pseudo-anomalias associadas a vales, nesta situação o conhecimento de campo torna-se fundamental para a modelagem.

Embora os minérios de ferro do Projeto Porteirinha apresentem baixos contrastes de densidade com suas encaixantes a modelagem empregada mostrou-se bastante eficiente na definição das camadas mineralizadas, bem como na previsão de zonas de mais alto teor e das modificações na geometria do minério em função dos processos de deformação tardios.

\section{Agradecimentos}

Os autores agradem a Vale S.A pela permissão da utilização dos dados aqui apresentados. 


\section{Referências}

Bekker, A.; Slack, J.F.; Planavsky, N., Krapež, B., Hofmann, A.; Konhauser, K.O.; Rouxel, O.J. 2010. Iron formation: the sedimentary product of a complex interplay among mantle, tectonic, oceanic, and biospheric processes. Econ. Geol., 105:467-508

Clout, J.M.F \& Simonson, B.M, 2005. Precambrian iron formations and iron-formation hosted iron ore deposits. In: Hedenquist , J.W , Thompson , J.F.H. , Goldfarb, R.J., Richards, J.R. (eds ) Economic Geology One Hundredth Anniversary Volume. p. 643-679.

Corrêia, C.R.A.; Cota, K.; Guimarães, M.F. 2009. Geologia e origem das mineralizações de ferro do depósito de Porteirinha (Grupo Macaúbas Neoproterozóico). CD ROM. Belo Horizonte. Vale S.A. $1^{\circ}$ Seminário de Geologia do Minério de Ferro.

CODEMIG 2006. Levantamento Aerogeofísico de Minas Gerais Área 8 - Minas Novas - Riacho dos Machados Espinosa, Relatório final do levantamento e processamento dos dados magnetométricos e gamaespectrométricos - Secretaria de Estado de Minas e Energia - SEME Governo de Minas Gerais, 2006

Karfunkel, J, Pedrosa-Soares, A.C. \& Dossin, I.A. 1985. O Grupo Macaúbas em Minas Gerais, revisão dos conhecimentos. Anais, $3^{\circ}$ Simp. Geol. de Minas Gerais, SBG-MG, Belo Horizonte, p. 45-59.

Klein, C. 1993. Sedimentology and geochemistry of the glaciogenic Late Proterozoic Rapitan Iron Formation in Canadá. Econ. Geol., 88:542-565.

Noce, C.M.; Pedrosa Soares, A.C.; Grossi Sad, J.H.; Baars, F.J.; Guimarães, M.L.V.; Mourão, M.A.A.; Oliveira, M.J.R.; Roque, N.C. 1997. Nova divisão estratigráfica regional do Grupo Macaúbas na Faixa Araçuaí: o registro de uma bacia neoproterozóica. Anais $9^{\circ} \mathrm{Simp}$. Geol. Minas Gerais, SBG - MG, Ouro Preto. Bol. 14: 29-31.

Pedrosa-Soares, A.C. \& Oliveira, M.J.R. 1997. Geologia da Folha Salinas. In: Grossi-Sad, J.H.; Lobato, L.M.; Pedrosa-Soares, A.C.; Soares-Filho, B.S. (eds). Projeto Espinhaço. CD-ROM. Belo Horizonte, CODEMIG, p. 419542.

Pedrosa-Soares, A.C.; Noce, C.M.; Alkmim, F.F.; Silva, L. C.; Babinski, M.; Cordani, U.; Castañeda, C. 2007. Orógeno Araçuaí: síntese do conhecimento 30 anos após Almeida 1977. Geonomos, 15(1):1-16.
Roque, N.C.; Grossi-Sad, J.H.; Noce, C.M.; Fonseca, E. 1997. Geologia da Folha Rio Pardo de Minas. In: GrossiSad, J.H.; Lobato, L.M.; Pedrosa-Soares, A.C.; SoaresFilho, B.S. (eds). Projeto Espinhaço. CD-ROM. Belo Horizonte, CODEMIG, p. 125 - 221.

Schobbenhaus, C. 1972a. Geologia da Serra do Espinhaço entre Porteirinha e Monte Azul, Norte de Minas Gerais. In: Schobbenhaus, C. 1972. Relatório Interno. Recife: SUDENE, 19 p.

Schobbenhaus, C. 1972b. Estudo geoeconômico preliminar do depósito de Ferro do Rio Peixe Bravo, Norte de Minas Gerais. Série Geologia Econômica, Recife. SUDENE, 8, 36 p.

Uhlein, A.; Trompette, R.; Alvarenga, C.J.S. 1999. Neoproterozoic glacial and gravitational sedimentation on a continental rifted margin: the Jequitaí-Macaúbas sequence (Minas Gerais, Brazil). Journal of South American Earth Science, 12: 435-451.

Vilela, O.V. 1986. As jazidas de minério de ferro dos municípios de Porteirinha, Rio Pardo de Minas, Riacho dos Machados e Grão Mogol, norte de Minas Gerais. In: Schobbenhaus, C.; Coelho, C.E.S. (Coords.). Principais depósitos minerais do Brasil. Brasília: DNPM. v.2, p. 111120.

Viveiros, J.M.F.; Sá, E.L.; Vilela, O.V.; Santos, O.M., Moreira, S.1978b. Prospecção das jazidas de minério de ferro dos municípios de Porteirinha, Rio Pardo de Minas, Riacho dos Machados e Grão Mogol, norte de Minas Gerais. In: Congr. Bras. de Geol., 30, 1978, Recife. Anais... Recife, 1978. v. 4, p. 1914-1923.

Viveiros, J.M.F.; Sá, E.L.; Vilela, O.V.; Santos, O.M.; Moreira, S.1978a. Geologia dos vales dos rios Peixe Bravo e Alto Vacaria, norte de Minas Gerais. In: Congresso Brasileiro de Geologia, 30., 1978, Recife. Anais... Recife, 1978. v. 1, p. 243-250.

Viveiros, J.M.F.; Sá, E.L.; Vilela, O.V.;Santos, O.M.; Moreira, S.; Neto, F.M.; Vieira, W.S.1979. Geologia dos vales dos Rios Peixe Bravo e Alto Vacaria, Norte de Minas Gerais. Atas 1. Simp. Geologia de Minas Gerais, SBG - Núcleo MG, Diamantina,.Bol. 1: 75-88.

Young, G.M., 1976. Iron formation and glaciogenic rocks of the Rapitan Group, Northwest Territories, Canadá. Precambrian Research, 3:137-158. 


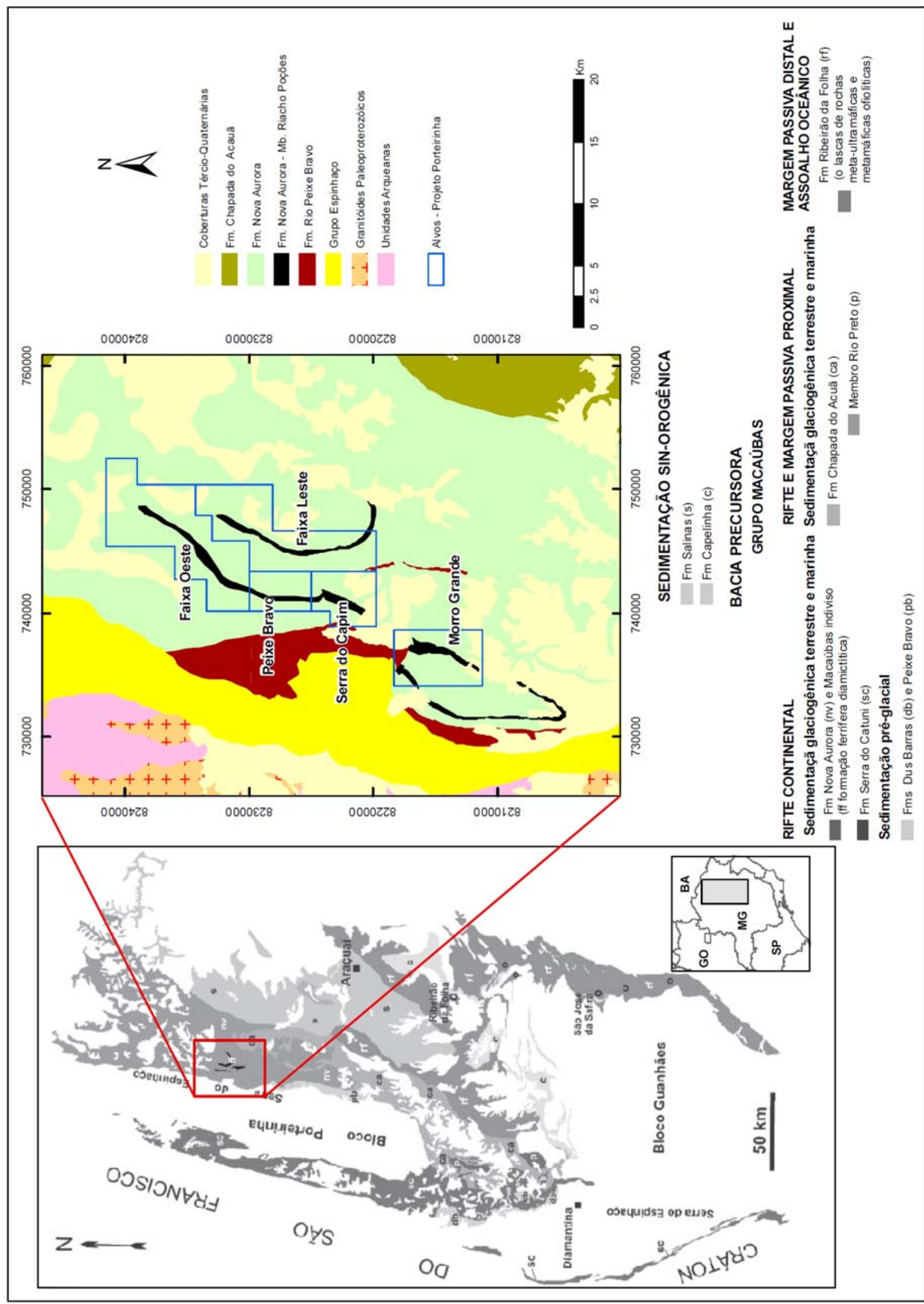

Figura 01 - Contexto geológico-geotectônico do Grupo Macaúbas no norte de Minas Gerais (Compilado de Viveiros et al. 1978a, Pedrosa-Soares et al. 2007). 
a)

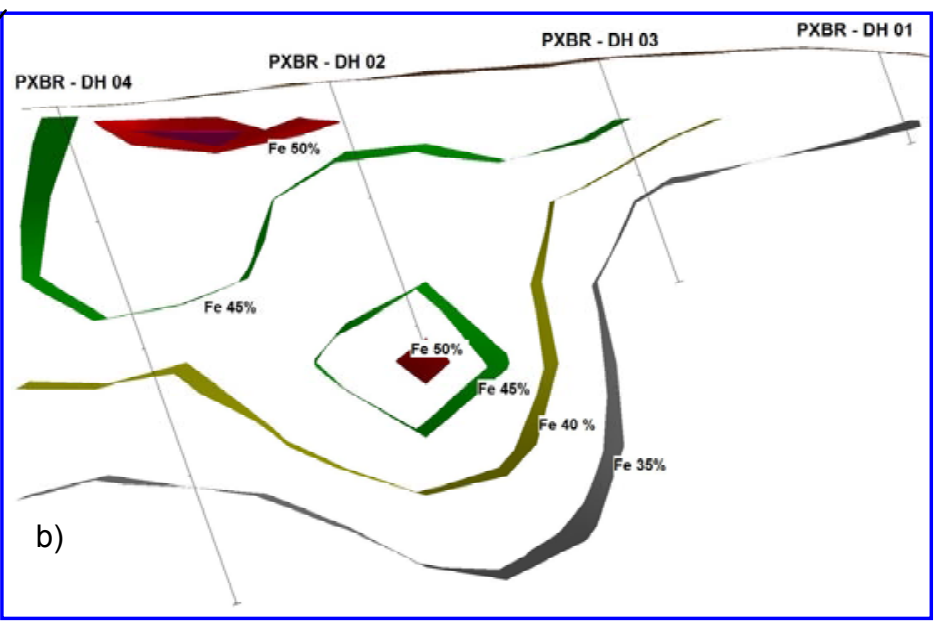

Figura 02 -. a) Modelagem 3D dos teores de ferro para o Alvo Peixe Bravo; b) Destaque para zona de alto teor em charneira sinclinal.

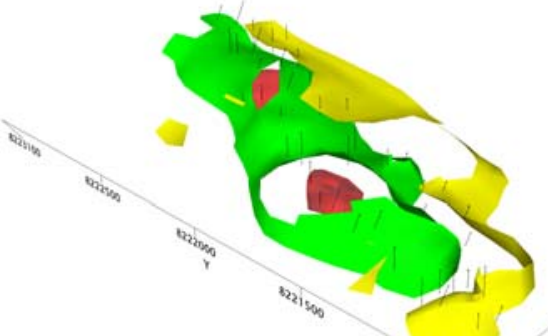

a)

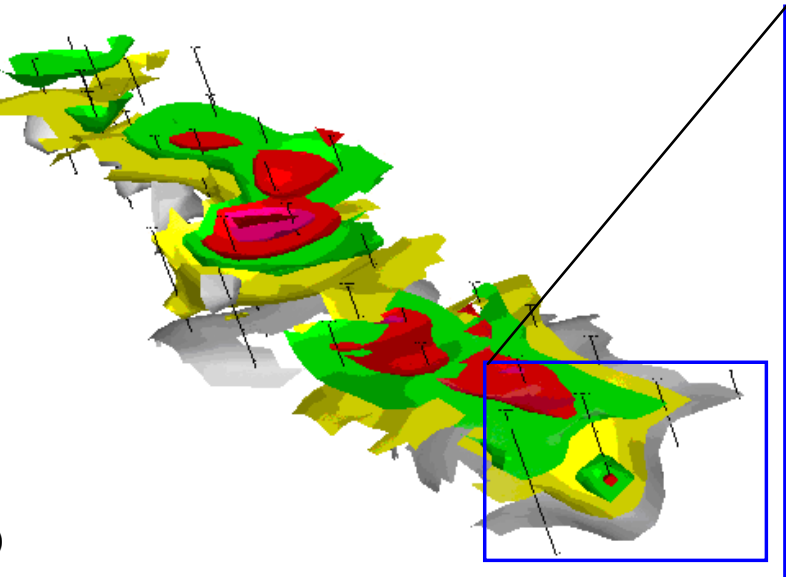
${ }^{2}{ }^{20} 0_{00}, p^{\circ}$
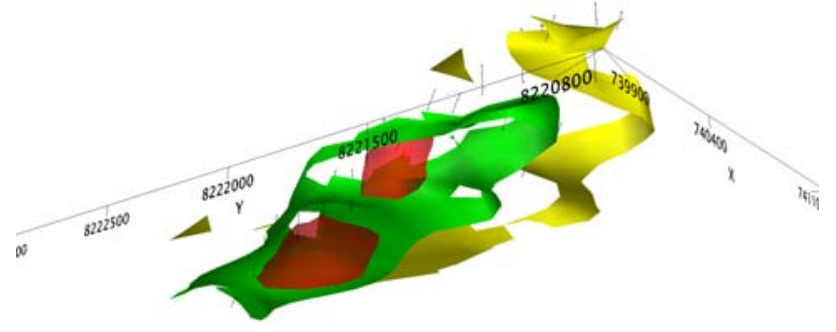

Figura 03 - Modelagem 3D dos dados de densidade para o Alvo Serra do Capim,em (a) com visada acima da topografia em (b) visada abaixo da topografia. Vermelho: $3,5 \mathrm{~g} / \mathrm{cm}^{3}$; Verde: $3,3 \mathrm{~g} / \mathrm{cm}^{3}$; Amarelo: $2,9 \mathrm{~g} / \mathrm{cm}^{3}$.

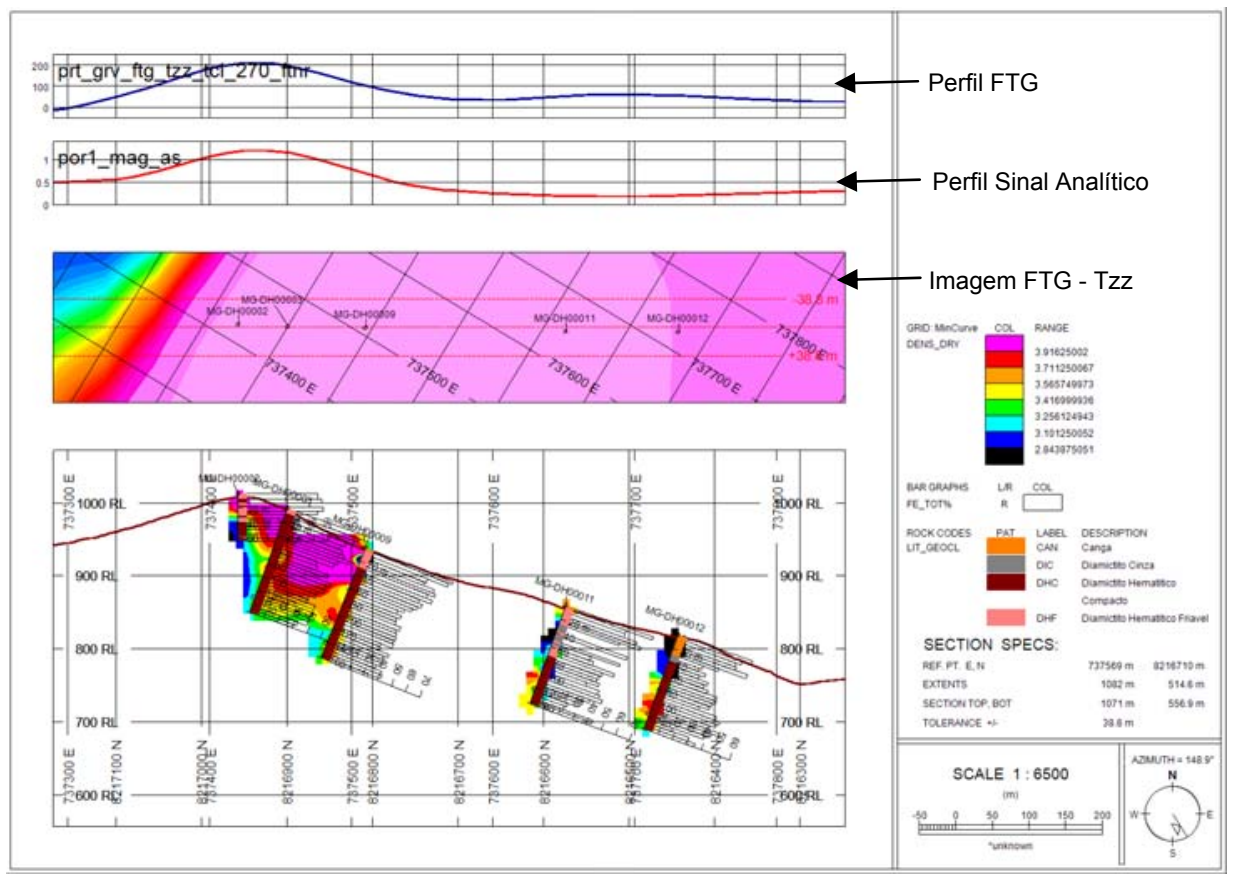

Figura 04 - Seção Morro Grande apresentando o produto de interpolação dos dados de densidade, os teores de ferro, litotipos e perfis gravimétricos e magnéticos. 
a) Peixe Bravo - LT $5400 \mathrm{~S}$

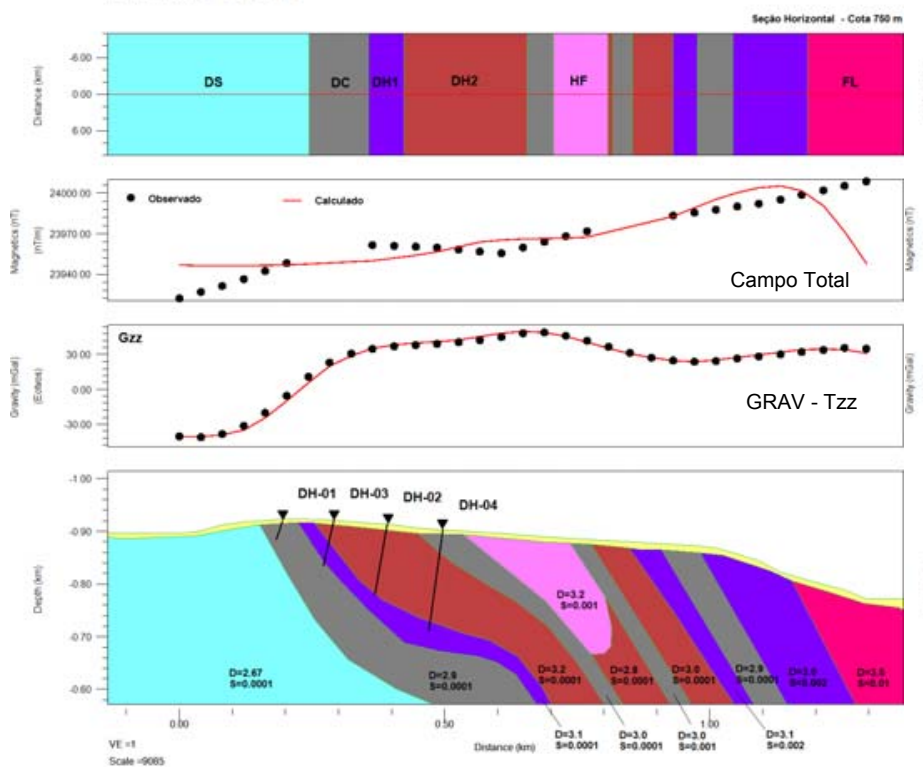

C) Serra do Capim - LT $9600 \mathrm{SW}$
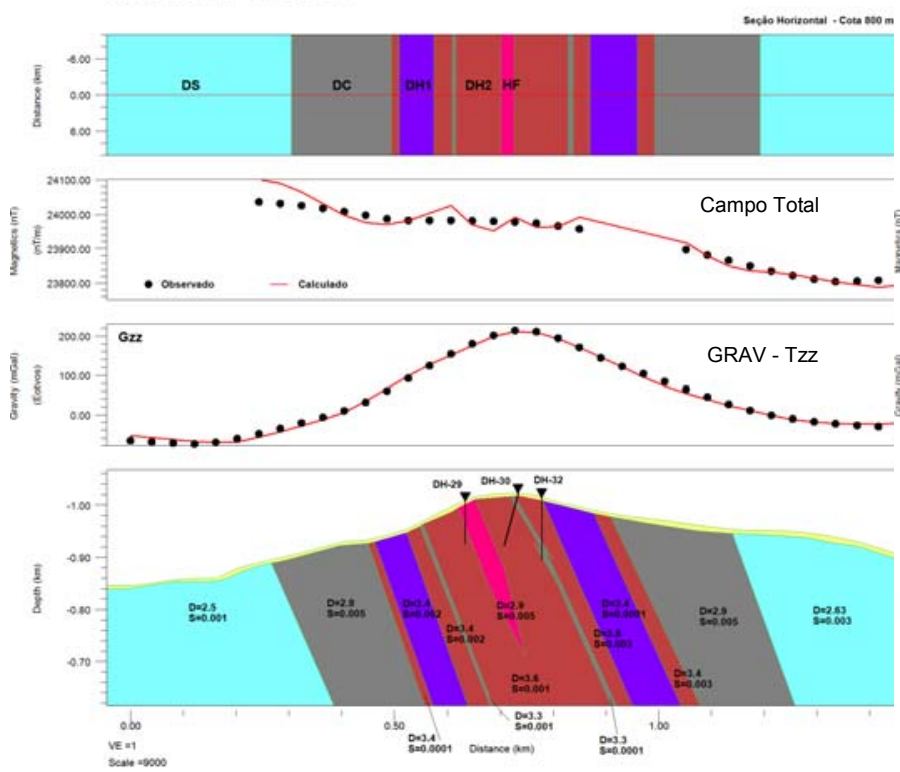

b) Peixe Bravo
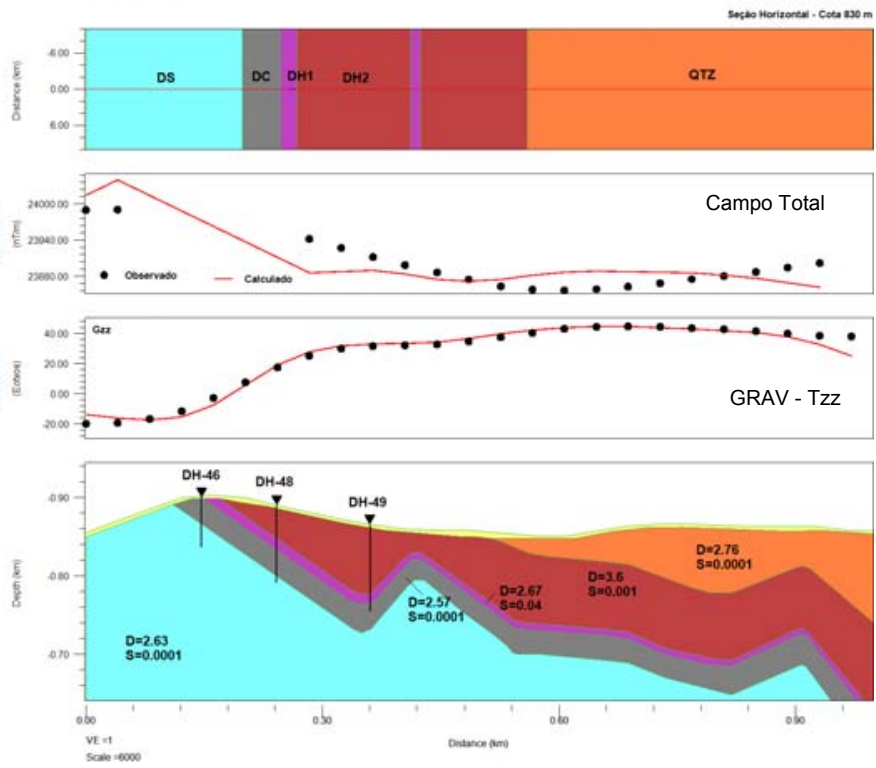

d) Morro Grande - LT $200 \mathrm{NE}$
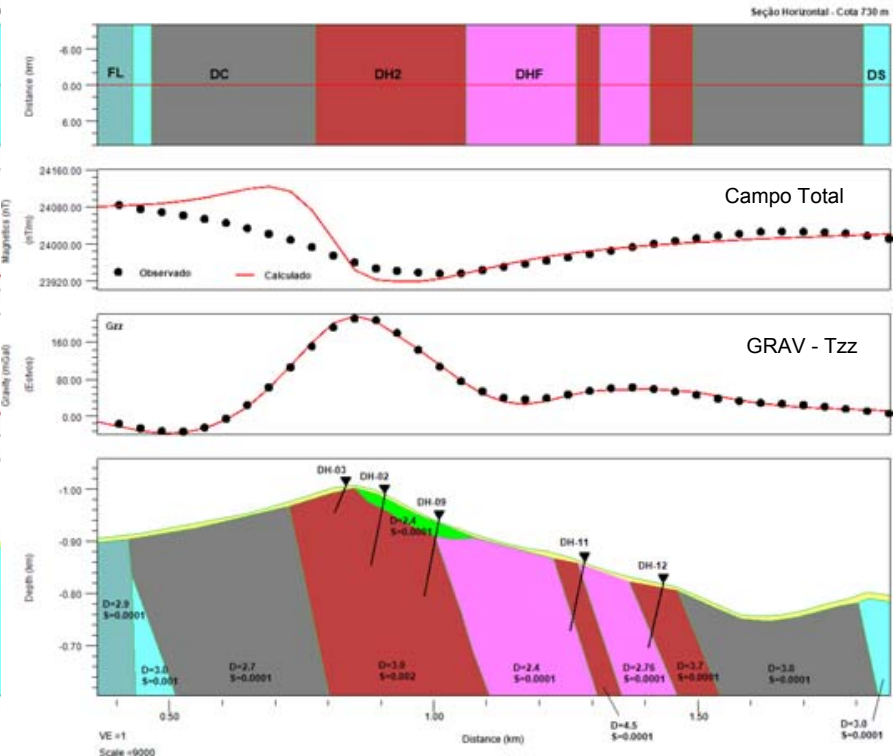

Figura 05 - Seções da modelagem gravimétrica dos alvos Peixe Bravo (a) e (b), Serra do Capim (c) e Morro Grande (d). Com indicação do ajuste entre as curvas observadas e modeladas dos dados gravimétricos e magnéticos. Atente que o modelamento foi executado com os dados gravimétricos e então para seções geradas foram atribuídas os valores de susceptibilidade magnética dos litotipos os quais produziram as curvas calculadas para este parâmetro. DS = Diamictito Sericítico; $D C=$ Diamictito Cinza; $D H 1=$ Diamictito Hematítico; $D H 2=$ Diamictito Hematítico; DHF = Diamictito Friável; HF = Hematita Friável; FL = Filito; $Q T Z=$ Quartizito/Metarritimito; $D=$ Densidade $\left(\mathrm{g} / \mathrm{cm}^{3}\right) ; S=$ Susceptibilidade magnética (SI). 